\title{
Management of patients with atopic dermatitis undergoing systemic therapy during COVID-19 pandemic in Italy: Data from the DA-COVID-19 registry
}

Andrea Chiricozzi $i^{1,2}$ | Marina Talamonti ${ }^{3}$ | Clara De Simone ${ }^{1,2} \mid$ Marco Galluzzo
Niccolò Gori
Concetta Fargnoli $^{26}$ | Andrea Carugno ${ }^{27}$ [ | Alberico Motolese ${ }^{28}$ | Franco Rongioletti ${ }^{29}$ | Paolo Amerio $^{30}$ | Riccardo Balestri ${ }^{31}$ | Concetta Potenza ${ }^{32}$ | Giuseppe Micali ${ }^{33}$ | Cataldo Patruno $^{34}$ | Iris Zalaudek ${ }^{35}$ | Maurizio Lombardo ${ }^{36}$ | Claudio Feliciani ${ }^{25}$ | Lucia Di Nardo ${ }^{1,2}$ | Fabrizio Guarneri ${ }^{37}$ | Ketty Peris ${ }^{1,2}$ | DA-COVID-19 study group*

${ }^{1}$ Dermatologia, Dipartimento Scienze Mediche e Chirurgiche, Fondazione Policlinico Universitario A. Gemelli IRCCS, Rome, Italy

${ }^{2}$ Dermatologia, Università Cattolica del Sacro Cuore, Rome, Italy

${ }^{3}$ Dermatology Unit, Policlinico Tor Vergata, Department of Systems Medicine, Tor Vergata University of Rome, Rome, Italy

${ }^{4}$ Section of Dermatology - Department of Clinical Medicine and Surgery, University of Naples Federico II, Naples, Italy

${ }^{5}$ Dermatology Unit, Fondazione IRCCS Ca, Granda Ospedale Maggiore Policlinico, Milan, Italy

${ }^{6}$ Department of Pathophysiology and Transplantation, UniversitàdegliStudi di Milano, Milan, Italy

${ }^{7}$ Section of Dermatology and Venereology, Department of Medicine, University of Verona, Verona, Italy

${ }^{8}$ Dermatological Clinic, Department of Clinical and Molecular Sciences, Polytechnic University of the Marche Region, Ancona, Italy

${ }^{9}$ Department of Dermatology, ASST Spedali Civili of Brescia, University of Brescia, Brescia, Italy

${ }^{10}$ Clinical Dermatology, San Gallicano Dermatological Institute, Rome, Italy

${ }^{11}$ Medical Sciences Department, Dermatologic Clinic, University of Turin, Turin, Italy

${ }^{12}$ Dermatology Section, Department of Medicine, University of Perugia, Perugia, Italy

${ }^{13}$ Department of Dermatology, University of Modena and Reggio Emilia, Modena, Italy

${ }^{14}$ Dermatology Unit, University of Campania Luigi Vanvitelli, Naples, Italy

${ }^{15}$ Dermatology UOC, Department of Experimental, Diagnostic and Specialty Medicine, University of Bologna, Bologna, Italy

${ }^{16}$ Department of Biomedical, Surgical and Dental Sciences, Clinical Dermatology, IRCCS Galeazzi Orthopaedic Institute, University of Milan, Milan, Italy

${ }^{17}$ Department of Dermatology, University of Pisa, Pisa, Italy

${ }^{18}$ Department of Health Sciences, Amedeo Avogadro University of Eastern Piedmont, Novara, Italy

${ }^{19}$ Dermatology Unit, Department of Medical, Surgical and Neurosciences, University of Siena, Siena, Italy

${ }^{20}$ Department of Biomedical Science and Human Oncology, Unit of Dermatology, University of Bari, Bari, Italy

${ }^{21}$ Dermatology Clinic, Department of Health Sciences, University of Florence, Florence, Italy

Abbreviations: AD, atopic dermatitis; COVID-19, coronavirus disease 2019; EASI, Eczema Area Severity Index; SARS-CoV-2, severe acute respiratory syndrome coronavirus type 2. 
${ }^{22}$ Dermatology Unit - Department of Medicine DIMED, University of Padova, Padova, Italy

${ }^{23}$ Section of Dermatology, Department of Health Promotion, Mother and Child Care, Internal Medicine and Medical Specialties, University of Palermo,

Palermo, Italy

${ }^{24}$ Unit of Dermatology, Department of Clinical Internal, Anesthesiological and Cardiovascular Sciences, Sapienza University of Rome, Rome, Italy

${ }^{25}$ Department of Medicine and Surgery, Section of Dermatology, University of Parma, Parma, Italy

${ }^{26}$ Dermatology, Department of Biotechnological and Applied Clinical Sciences, University of L'Aquila, L'Aquila, Italy

${ }^{27}$ Dermatology Unit, ASST Papa Giovanni XXIII Hospital, Bergamo, Italy

${ }^{28}$ Dermatology Unit, Department of MedicalSpecialties, Arcispedale Santa Maria Nuova-IRCCS di Reggio Emilia, Reggio Emilia, Italy

${ }^{29}$ Vita-Salute San Raffaele University and IRCCS San Raffaele Hospital, Milan, Italy

${ }^{30}$ Department of Medicine and Aging Science, Dermatologic Clinic, G. D'Annunzio University, Chieti, Italy

${ }^{31}$ Division of Dermatology, Santa Chiara Hospital, Trento, Italy

${ }^{32}$ Department of Medico-Surgical Sciences and Biotechnologies, Dermatology Unit, Daniele Innocenzi, Sapienza, University of Rome - Polo Pontino, Rome, Italy

${ }^{33}$ Dermatology Clinic, University of Catania, Catania, Italy

${ }^{34}$ Dermatology Unit, Department of Health Sciences, Università Magna Graecia, Catanzaro, Italy

${ }^{35}$ Department of Dermatology, University of Trieste, Trieste, Italy

${ }^{36}$ Unit of DermatologicalDiseases, ASST Sette Laghi, Ospedale di Circolo, Varese, Italy

${ }^{37}$ Dermatology, Department of Clinical and Experimental Medicine, University of Messina, Messina, Italy

\section{Correspondence}

Andrea Chiricozzi, Institute of

Dermatology, Catholic University of the

Sacred Heart - Policlinico Universitario

A. Gemelli IRCCS Foundation, Rome,

Largo Agostino Gemelli 8, 00168 Rome,

Italy.

Email: chiricozziandrea@gmail.com

\section{Abstract}

Background: Few and small studies have described the management of immunomodulant/immunosuppressive therapies or phototherapy in atopic dermatitis (AD) patients during coronavirus disease 2019 (COVID-19) pandemic.

Methods: A national registry, named DA-COVID-19 and involving 35 Italian dermatology units, was established in order to evaluate the impact of COVID-19 pandemic on the management of adult $A D$ patients treated with systemic immunomodulant/immunosuppressive medications or phototherapy. Demographic and clinical data were obtained at different timepoints by teledermatology during COVID-19 pandemic, when regular visits were not allowed due to sanitary restrictions. Disease severity was assessed by both physician- and patient-reported assessment scores evaluating itch intensity, sleep disturbances, and AD severity.

Results: A total of 1831 patients were included, with 1580/1831 (86.3\%) continuing therapy during pandemic. Most patients were treated with dupilumab $(86.1 \%$, $1576 / 1831)$ that was interrupted in only $9.9 \%(156 / 1576)$ of cases, while systemic immunosuppressive compounds were more frequently withdrawn. Treatment interruption was due to decision of the patient, general practitioner, or dermatologist in $39.9 \%$ (114/286), 5.6\% (16/286), and 30.1\% (86/286) of cases, respectively. Fear of increased susceptibility to SARS-CoV-2 infection $(24.8 \%, 71 / 286)$ was one of the main causes of interruption. Sixteen patients $(0.9 \%)$ resulted positive to SARS-CoV-2 infection; 3 of them $(0.2 \%)$ were hospitalized but no cases of COVID-related death occurred.

Conclusions: Most AD patients continued systemic treatments during COVID pandemic and lockdown period, without high impact on disease control, particularly dupilumab-treated patients.

\section{KEYWORDS}

atopic dermatitis, COVID, SARS-CoV 


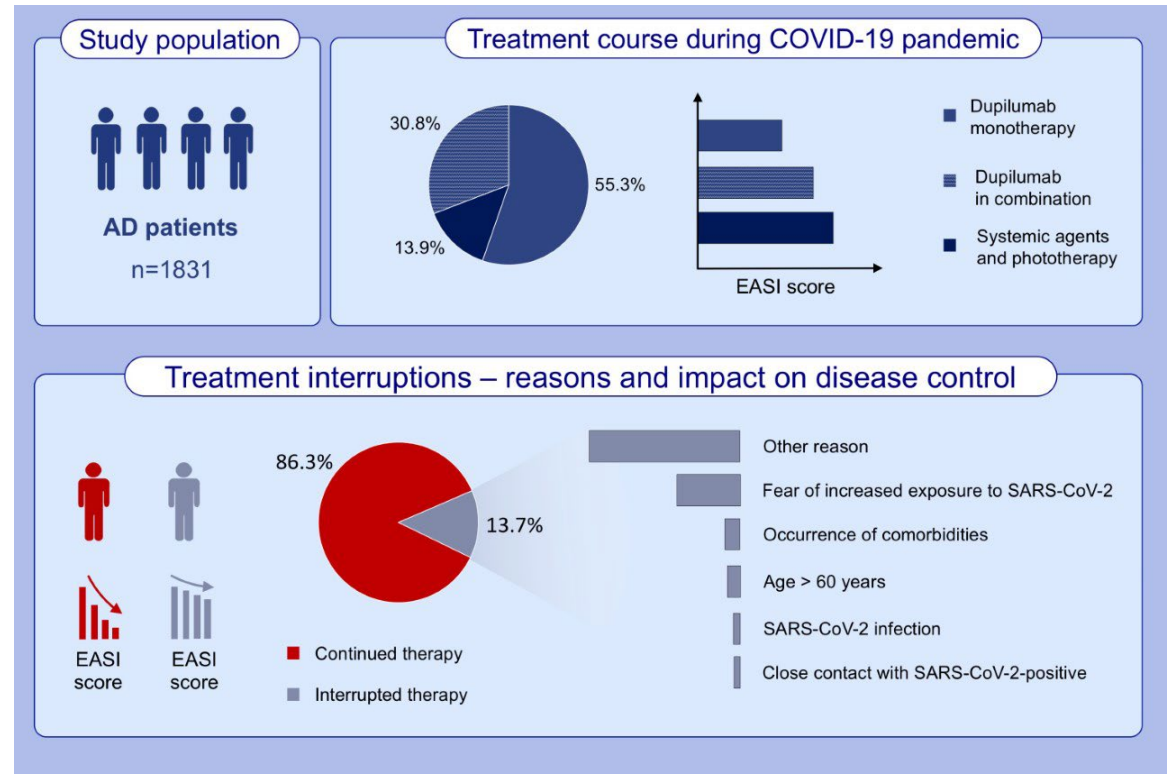

\section{GRAPHICAL ABSTRACT}

Among 1831 studied AD patients, 86.1\% were treated with dupilumab. Patients continuing therapy experienced a marked reduction of disease severity during pandemic. The causes of treatment interruption included: fear of increased susceptibility to SARS-CoV-2 infection (24.8\%), occurrence of comorbidities (5.9\%), age above 60 years (5.2\%), SARS-CoV-2 infection (2.8\%), close contact with SARS-CoV-2positive subject (2.4\%), other reasons, for example, inability to maintain drug supply, non-medical/unspecified causes (58.7\%).

\section{INTRODUCTION}

COVID-19, caused by SARS-CoV-2 infection, has spread rapidly worldwide becoming pandemic, as defined by the World Health Organization on 18th March 2020. ${ }^{1}$ Most patients exhibit mild-to-moderate symptoms and recover without sequelae, though hospitalization, generally due to pneumonia, and more severe respiratory involvement such as acute respiratory distress syndrome, septic shock, and/or multiple organ failure, associated with high mortality, may occur. ${ }^{1}$

Italy has faced the first wave of SARS-CoV-2 infection out of China before the rapid worldwide pandemic spreading. To face the virus spreading, a nationwide lockdown period (phase I) limiting all kind of activities including healthcare services was decided on March 10th and lasted until May 4th, when a phase II was planned with a gradual re-opening of hospital dermatology services. During these two initial phases, medical visits were restricted to urgent cases, and the use of teledermatology was implemented in many dermatological services. On 15th June 2020, a phase III was established recovering almost all activities with sanitary restrictions, and healthcare services were restored based on the decision of local sanitary authorities.

Thereby, COVID-19 pandemic led to the sudden need of increasing the use of web and phone consulting, and defining practical guidelines for the management of immune-mediated dermatologic conditions, such as $A D$ that in moderate-to-severe cases are commonly treated with systemic immunomodulant/immunosuppressive compounds or phototherapy. The effect of immunomodulant/immunosuppressive compounds on the clinical course of COVID-19 is currently unclear, and there is concern of an increased risk of infection in AD patients treated with systemic compounds, though the continuation of therapy during pandemic was recommended by national and international scientific societies. ${ }^{2-7}$ Nevertheless, immunomodulant/immunosuppressive agents, such as methotrexate, mycophenolate, azathioprine, and cyclosporine, were suggested to be tapered to the lowest effective dose, likely avoiding disease flare, and to consider drug discontinuation in patients when viral symptoms are present. ${ }^{2,5}$ Similarly, caution was recommended in prescribing systemic corticosteroids given their broad immunosuppressive effects. ${ }^{2,5}$ Furthermore, some authors recommended halting office-based phototherapy to minimize potential exposure to SARS-CoV-2 virus and instead encourage exposure of affected areas to natural sunlight, bleach baths, and wet wraps. ${ }^{5}$ However, current recommendations are based on limited knowledge regarding the risk of systemic immunomodulant/immunosuppressive compound use, and few data related to $A D$ patients treated during COVID-19 pandemic.

We designed a national registry, the DA-COVID-19 registry, aimed to evaluate the impact of the pandemic on the therapeutic management and clinical course of $A D$ in patients treated with any systemic immunomodulant/immunosuppressive compound or phototherapy. This observational study analyzed clinical and demographic characteristics of moderate-to-severe AD patients, who were managed with telemedicine and eventually by regular ambulatory visits during the COVID-19 pandemic.

\section{2 | METHODS}

This cross-sectional, multicentric, observational study was conducted in 35 Italian centers. This registry, which was aimed to collect data on moderate-severe $A D$ patients treated with systemic agents and/or phototherapy during COVID-19 outbreak, has 
been promoted by the Italian Society of Dermatology (SIDeMaST) and approved by the national ethical committee for COVID-19related studies (Istituto Nazionale per le Malattielnfettive Lazzaro Spallanzani I.R.C.C.S.). The study period included the three phases of first-wave COVID-19 pandemic in Italy (Figure S1).

Adult patients (aged $\geq 18$ years) affected by moderate-to-severe $A D$, treated with systemic immunosuppressive/immunomodulant compounds or phototherapy, were included in the DA-COVID-19 registry if face-to-face evaluation or remote visit (via telephone or web consulting) were performed between 10th March and 30th April 2020. By April 30th, data have been collected monthly, thereafter, on an ad hoc database. Data were collected at 3 different timepoints: April 30th (Timepoint 1), May 30th (Timepoint 2), and June 30th (Timepoint 3) (Figure S1). Subjects who signed the informed consent were included in this study. Baseline data included age, gender, occupation, atopic comorbidities, smoking habits (smoker, former smoker, or non-smoker), and disease severity.

\section{1 | Disease severity assessment}

Disease severity was assessed by EASI score at timepoint 1 (either assessed during face-to-face visit or the last recorded EASI score in patient's file) and at timepoint 3 , being performed if dermatology units restored their regular outpatient clinical activity. At timepoint 2, due to sanitary restriction, no EASI score was reported. In addition, patient-reported evaluations included: 0-10 NRS for pruritus intensity (itch NRS), sleep disturbances/sleeplessness by a 0-10 NRS scale (sleep NRS), self-evaluated AD severity by a 0-10 NRS scale (AD-NRS), self-evaluation of patient's $A D$ clinical course (patient perception of "AD status," defined as stable/no flaring, improved, or worsened, during the observation period), and ongoing treatment. Details about treatment interruption or suspension were recorded. Data on SARS-CoV-2 swab testing, hospitalization, clinical outcomes of COVID-19 disease, and quarantine due to close contact to COVID-9 patients were also collected.

\section{2 | Statistical analysis}

Patients were analyzed according to their ongoing therapy to identify possible differences in any of the demographic or clinical variables collected. Frequency and percentages were the descriptive analyses performed on the categorical variables. Continuous variables were summarized as means \pm standard deviation. For categorical variables, differences between groups were evaluated using chi-squared test or Fisher's exact test (if more than $20 \%$ of the cells in a contingency table have expected counts less than 5). For quantitative variables, the Shapiro-Wilk test was performed in order to test the normality of data. If the $p$-value was less than or equal to 0.05 (non-normality), the comparison between groups was performed by means of the non-parametric Wilcoxon rank-sum test. Otherwise, the comparison was performed using the t-test. Moreover, comparison between timepoint 1 value and the other timepoints was performed using the paired t-test (or the Wilcoxon signed-rank test in the case of non-normal data). Finally, an ANOVA test (or Kruskal-Wallis test in the case of non-normal data) was performed to compare the means in case of more than 2 groups. Differences were considered statistically different if $P$ values resulted $<0.05$. Analyses were performed using software SAS 9.4 version (SAS, NC, USA).

\section{3 | RESULTS}

The DA-COVID registry included 1831 patients with moderate-tosevere AD presenting demographic and clinical characteristics as illustrated in Table 1. Overall, 142/1831 (7.7\%) patients were lost to follow-up throughout the observation period.

\section{1 | SARS-CoV-2 infection in the study population}

Fifty-nine of 1831 (3.2\%) AD patients performed in total 79 SARSCoV-2 nasal-throat swab tests; 16/1831 (0.9\%) had a confirmed diagnosis of SARS-CoV-2 infection; and $3(0.2 \%)$ were hospitalized. No cases of death from COVID-related disease occurred in our study population throughout the whole observation period. The 16 SARS-CoV-2-positive patients had a mean age of 45.1 years $( \pm 16.4)$; 9 were females $(56.3 \%)$, presenting rhinitis as the most common atopic comorbidity 10/16 (62.5\%) (Table 2). AD severity was in line with the overall patient population (data not shown). Comparing AD patients positive to SARS-CoV-2 nasal-throat swab testing with those who resulted negative, no significant difference in mean baseline EASI score was found (7.744 \pm 2.062 for SARS-CoV-2-positive patients vs $5.830 \pm 0.9865$ for SARS-CoV2-negative patients, $P=0.3577)$. Fifteen of 16 (93.8\%) patients were undergoing dupilumab therapy when SARS-CoV-2 occurred (Table 2). Dupilumab was mostly used in monotherapy (80\%, 13/15) while in $20.0 \%$ (3/15) of patients was combined with systemic corticosteroids and/or methotrexate. Half of SARS-CoV-2-positive patients discontinued treatment. SARS-CoV-2-positive patients who continued treatment were all undergoing dupilumab therapy and exhibited a significant reduction of mean EASI score from timepoint 1 to timepoint $3(7.7 \pm 2.1$ at timepoint 1 vs $2.3 \pm 1.3$ at timepoint 3 $P=0.0468$ ). Similarly, EASI score significantly decreased in patients discontinuing therapy overtime (timepoint 1: $9.9 \pm 9.5$ vs timepoint 3: $0.4 \pm 0.52, P=0.013)$. Neither dupilumab-related adverse events (i.e., injection site reaction or conjunctivitis) nor COVID-19 complication or worsening was reported in those cases continuing therapy. Because of close contact with COVID-19 cases or high-risk conditions for SARS-CoV-2 infection, 3.2\% (58/1831) of patients underwent quarantine. 
TAB LE 1 Clinical and demographic characteristics of patients included in the DA-COVID-19 registry, dissecting patients who continued or discontinued therapy as subcohorts

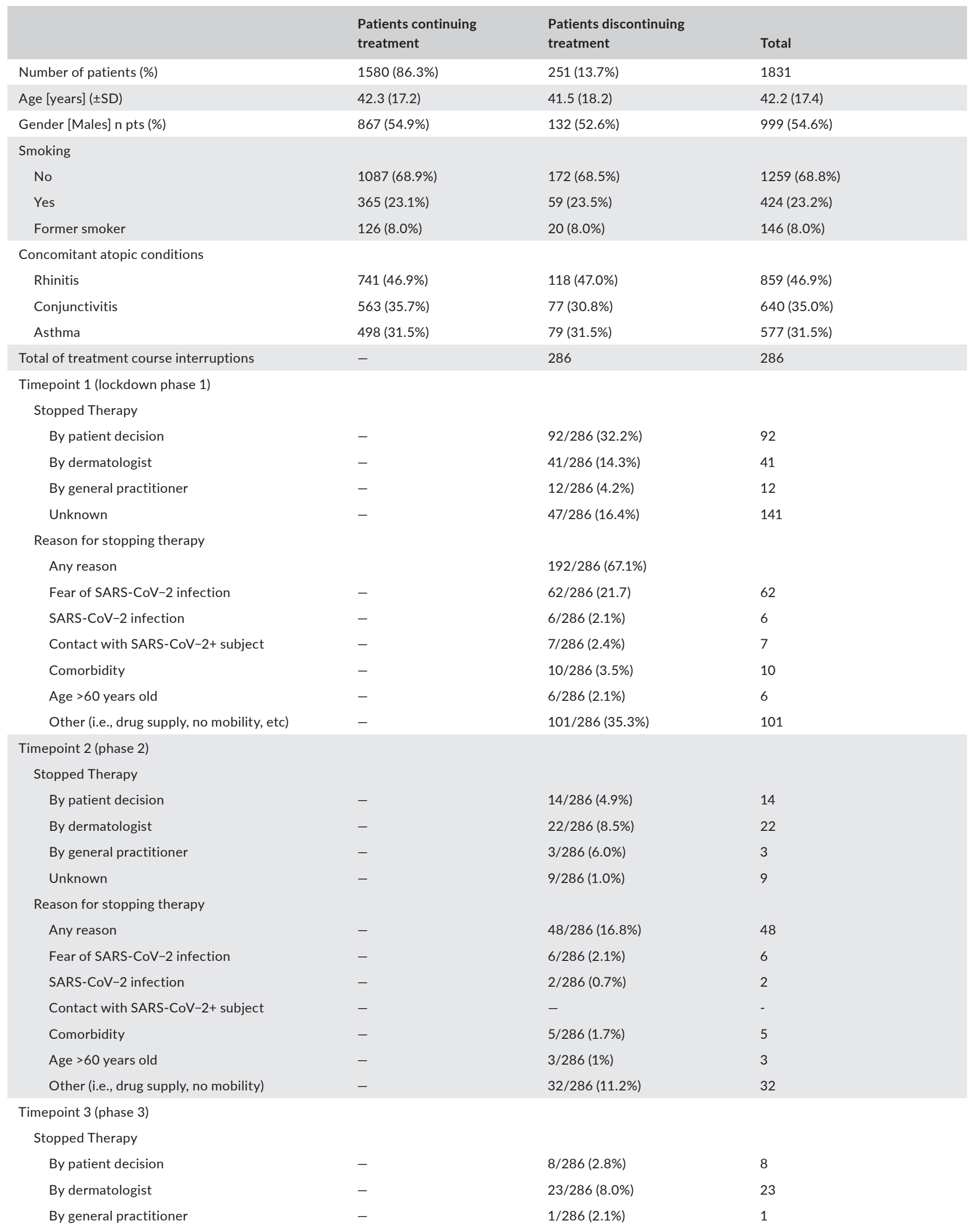


WILEY-Allergy

TABLE 1 (Continued)

\begin{tabular}{|c|c|c|c|}
\hline & $\begin{array}{l}\text { Patients continuing } \\
\text { treatment }\end{array}$ & $\begin{array}{l}\text { Patients discontinuing } \\
\text { treatment }\end{array}$ & Total \\
\hline Unknown & - & $14 / 286(30.4 \%)$ & 14 \\
\hline Any reason & - & $46 / 286(16.1 \%)$ & 46 \\
\hline Fear of SARS-CoV-2 infection & - & $3 / 286(1.0 \%)$ & 3 \\
\hline Comorbidity & - & $2 / 286(0.7 \%)$ & 2 \\
\hline Age $>60$ years old & - & $6 / 286(2.1 \%)$ & 6 \\
\hline Other (i.e., drug supply, no mobility) & - & $35 / 286(12.2 \%)$ & 35 \\
\hline $\begin{array}{l}\text { Missing data about decision of treatment } \\
\text { interruption }\end{array}$ & & & $70 / 286(24.5 \%)$ \\
\hline
\end{tabular}

Data are reported as means ( \pm standard deviation) or numbers (\%).

TABLE 2 Clinical and demographic data of COVID-19+ AD patients

\begin{tabular}{|c|c|c|c|}
\hline & $\begin{array}{l}\text { Patients } \\
\text { continuing } \\
\text { treatment }\end{array}$ & $\begin{array}{l}\text { Patients discontinuing } \\
\text { treatment }\end{array}$ & Total=1831 \\
\hline Number of patients & 8 & 8 & 16 \\
\hline Sex M/F (\%/\%) & $2 / 6(25 \% / 75 \%)$ & $5 / 3(62.5 \% / 37.5 \%)$ & $7 / 9$ (43.8\%/56.2\%) \\
\hline Age ( \pm SD) & $47.9( \pm 17.7)$ & $42.3( \pm 15.7)$ & $45.1( \pm 16.4)$ \\
\hline Rhinitis & $6(75.0 \%)$ & $4(50.0 \%)$ & $10(62.5 \%)$ \\
\hline Conjunctivitis & 4 (50.0\%) & 4 (50.0\%) & 8 (50.0\%) \\
\hline Asthma & $3(37.5 \%)$ & $4(50 \%)$ & $7(43.8 \%)$ \\
\hline Hospitalization & $1(12.5 \%)$ & $2(25.0 \%)$ & 3 (18.8\%) \\
\hline \multicolumn{4}{|l|}{ Therapy } \\
\hline $\begin{array}{l}\text { Dupilumab n. } \\
\text { patients (\%) }\end{array}$ & $8(100.0 \%)$ & 7 (87.5\%) & 15 (93.8\%) \\
\hline Antihistamines & 0 & $3(37.5 \%)$ & 3 (18.8\%) \\
\hline $\begin{array}{c}\text { Corticosteroids n. } \\
\text { patients (\%) }\end{array}$ & 0 & $2(25.0 \%)$ & 2 (12.5\%) \\
\hline $\begin{array}{l}\text { Phototherapy n. } \\
\text { patients (\%) }\end{array}$ & 0 & $2(25.0 \%)$ & $2(12.5 \%)$ \\
\hline $\begin{array}{c}\text { Methotrexate } \mathrm{n} . \\
\text { patients (\%) }\end{array}$ & $1(12.5 \%)$ & 0 & $1(6.3 \%)$ \\
\hline
\end{tabular}

Data are reported as means ( \pm standard deviation) or numbers (\%).

\subsection{Characterization of treatment path in the study population}

Overall, 63.2\% (1157/1831) and 36.8\% (674/1831) of patients were treated in monotherapy or with two or more systemic agents, respectively. Most patients (55.3\%, 1013/1576) were treated with dupilumab monotherapy, while in $30.8 \%$ (563/1831) dupilumab was associated with other systemic agents or phototherapy (Figure S2). Immunosuppressive systemic compounds were used as either monotherapy or combination therapy as showed in Table 3. More frequently, antihistamines (76.2\%, 429/563), oral corticosteroids (19.2\%, 108/563), and cyclosporine (12.6\%, 71/563) were associated with dupilumab as combination therapy. A small percentage of AD patients $(13.9 \%, 255 / 1831)$ was exclusively treated with conventional systemic therapies, including phototherapy (Figure S2). Notably, patients treated with dupilumab combined with other systemic therapies had significantly higher rates of concomitant atopic conditions compared to patients treated with dupilumab monotherapy or systemic immunosuppressive compounds $(P<0.001$; Table S1). Across treatment subgroups, no worsening of atopic comorbid conditions was reported as adverse event.

In a small proportion of patients (53/1831, 2.9\%), systemic therapy was modified including a total of 66 therapy modifications consisting of drug dosage adjustment (i.e., tapering down or increasing 
dose) or lengthening drug administration interval, at least once. The addition to or substitution of the systemic therapy with topical agents, homeopathy, or other non-systemic therapies (i.e., sun exposure) occurred in 937 cases.

In total, 251 patients discontinued treatment. One hundred ten of 251 patients (43.8\%) temporarily suspended therapy that was restarted during the whole observation period, whereas 141 patients continued to manage AD with topical therapies, emollients, homeopathy, or other non-systemic therapies.

\section{3 | Different management of immunosuppressive systemic compounds compared to dupilumab}

The majority of patients $(86.3 \%, 1580 / 1831)$ continued therapy, whereas $13.7 \%$ of patients $(251 / 1831)$ withdrew systemic therapy at least once, with a mean duration of treatment interruption of 56.5 days ( \pm 27.2 ), and a total number of therapeutic course interruptions of 286. Most of treatment interruptions was recorded at timepoint 1 (67.1\%, 192/286), whereas in $16.8 \%(48 / 286)$ and $16.2 \%$ $(46 / 286)$ of cases, therapy was withdrawn at timepoint 2 and 3 , respectively. Treatment interruptions occurred with similar distribution across the three cohorts of patients treated with systemic immunosuppressive compounds ( $36.4 \%$ of cases with at least one treatment interruption), dupilumab monotherapy (32.9\%), or dupilumab combined with other systemic therapies (30.7\%). Nevertheless, considering the rate of treatment interruption for each drug, dupilumab was interrupted in only $9.9 \%(156 / 1576)$ of cases, whereas cyclosporine, antihistamines, oral corticosteroids, phototherapy, and methotrexate were interrupted in 40.9\% (52/127), 39.9\% (190/476), $23.4 \%$ (34/145), 74.1\% (60/81), and 23.5\% (12/51) of cases, respectively (Table 3).

In $39.9 \%(114 / 286)$ of cases, treatment interruption was due to patient decision, while in $5.6 \%(16 / 286)$ and $30.1 \%(86 / 286)$ of cases, treatment interruption was suggested by the general practitioner and by the dermatologist, respectively. In particular, the interruption of systemic immunosuppressive compounds was more frequently suggested by the dermatologist $(40.4 \%, 42 / 104)$, whereas dupilumab monotherapy or dupilumab combined with other systemic therapies were mostly interrupted because of patient decision (53.2\% [50/94]; 50\% [44/88], respectively) (Table S1). In details, one or more reasons led to the decision of stopping therapy: (i) the inability to maintain drug supply, other non-medical or unspecified causes (58.7\%, 168/286 cases); (ii) the occurrence of concomitant comorbid conditions (5.9\%, 17/286 cases); (iii) age, over 60 years old (5.2\%, 15/286 cases); (iv) close contact with SARS-CoV-2+ subject (2.4\%, 7 /286); (v) SARS-CoV-2 infection (2.8\%, 8/286); and (vi) fear of increased susceptibility to SARS-CoV-2 infection (24.8\%, 71/286). Fear of increased susceptibility to SARS-CoV-2 infection caused treatment interruption in $23.4 \%, 23.9 \%$, and $26.9 \%$ of patients treated with dupilumab monotherapy, dupilumab combined with other systemic therapies, and systemic immunosuppressive compounds, respectively. 


\subsection{Different AD clinical courses in patients withdrawing treatment compared to patients continuing therapy}

At timepoint 1 (lockdown phase), disease severity assessment of the whole patient population showed: mean EASI score of $6.8 \pm 7.7$, itch NRS of $2.6 \pm 2.2$, sleep NRS of $1.7 \pm 2.1$, and self-assessment of AD severity, AD-NRS of $2.5 \pm 2.1$ (Table 4). During the study period, patients experienced a significant reduction of mean itch NRS, mean sleep NRS, and mean AD-NRS scores, achieving lower mean scores at timepoint 3, compared to timepoint 1 (Tables S2 and S3). This improvement reflected the significant decrease of mean EASI score at timepoint $3(3.4 \pm 4.4)$ compared to timepoint 1 (6.8 \pm 7.7, $P<0.0001)$. Reduction of mean EASI score was observed in both patients continuing treatment and patients interrupting systemic therapy, though at different extent (Table 4). Indeed, mean EASI score changed in the cohort of patients continuing treatment over time (6.6 \pm 7.8 at timepoint 1 vs $2.8 \pm 3.4$ at timepoint 3$)$, obtaining

TAB LE 4 Disease severity assessed at different timepoints related to therapy continuation or discontinuation. Both patient-assessed severity measurements-itch NRS score, sleep NRS score, AD-NRS score, course of disease (improved, stable, worsened)-and physicianassessed severity measure (EASI score) were performed in all patient population, in the subcohort of patients treated continuously, and in the subcohort of patients who discontinued treatment

\begin{tabular}{|c|c|c|c|}
\hline & $\begin{array}{l}\text { Patients continuing } \\
\text { treatment (n. pts: 1580) }\end{array}$ & $\begin{array}{l}\text { Patients discontinuing } \\
\text { treatment (n. pts: } 251 \text { ) }\end{array}$ & $\begin{array}{l}\text { Total population } \\
\text { (n. pts: 1831) }\end{array}$ \\
\hline \multicolumn{4}{|l|}{ Timepoint 1 (lockdown- phase 1) } \\
\hline Mean itch NRS score $( \pm S D)$ & $2.4(2.1)$ & $3.7(2.3)$ & $2.6(2.2)$ \\
\hline Mean sleep NRS score $( \pm S D)$ & $1.6(2.0)$ & $2.7(2.4)$ & $1.7(2.1)$ \\
\hline \multicolumn{4}{|l|}{ Self-reported AD status ${ }^{\S}$} \\
\hline Improved n. pts (\%) & $454(28.8 \%)$ & 39 (15.5\%) & $493(27.0 \%)$ \\
\hline Stable n. pts (\%) & 961 (60.9\%) & $122(48.6 \%)$ & $1083(59.2 \%)$ \\
\hline Worsened n. pts (\%) & $162(10.3 \%)$ & $90(35.9 \%)$ & $252(13.8 \%)$ \\
\hline \multicolumn{4}{|l|}{ Timepoint 2 (phase 2) } \\
\hline AD-NRS score $( \pm S D)$ & $2.1(1.9)$ & $3.7(2.6)$ & $2.3(2.1)$ \\
\hline \multicolumn{4}{|l|}{ Self-reported AD status ${ }^{\S}$} \\
\hline Improved n. pts (\%) & $417(27.2 \%)$ & $53(22.1 \%)$ & $470(26.5 \%)$ \\
\hline Stable n. pts (\%) & $980(63.8 \%)$ & 102 (42.5\%) & $1082(61.0 \%)$ \\
\hline Worsened n. pts (\%) & $138(9.0 \%)$ & 85 (35.4\%) & $223(12.6 \%)$ \\
\hline \multicolumn{4}{|l|}{ Timepoint 3 (phase 3) } \\
\hline Mean EASI score $( \pm S D)^{\complement}$ & $2.8(3.4)$ & $7.3(7.7)$ & $3.4(4.4)$ \\
\hline Mean itch NRS score $( \pm S D)$ & $3.3(2.6)$ & $3.3(2.6)$ & $2.2(2.1)$ \\
\hline Mean sleep NRS score $( \pm S D)$ & $1.2(1.7)$ & $2.2(2.4)$ & $1.3(1.9)$ \\
\hline Change in itch NRS from timepoint 1 to timepoint 3 & $-0.3(2.0)$ & $-0.2(2.7)$ & $-0.3(2.1)^{*}$ \\
\hline Change in sleep NRS from timepoint 1 to timepoint 3 & $-0.3(1.9)$ & $-0.3(2.8)$ & $-0.3(2.0)^{*}$ \\
\hline Change in AD-NRS from timepoint 1 to timepoint 3 & $-0.4(1.8)$ & $-0.3(2.4)$ & $-0.4(1.9)^{*}$ \\
\hline
\end{tabular}

Abbreviations: AD, atopic dermatitis; EASI: Eczema Area and Severity Index; NRS: Numeric Rating Scale; pts: patients; SD: standard deviation. $\# P<0.001$, Wilcoxon rank-sum test was used to compare the 2 patient subcohorts at timepoint 1.

$\S P<0.001$, chi-squared test was used for statistical analysis.

çMean EASI score was calculated on 1831 and 746 patients at timepoint 1 and 3, respectively.

${ }^{*} P<0.0001$, paired $s$ test was used to compare T1 vs T3 in the total population. 
a 10-fold higher reduction compared to the cohort of patients withdrawing treatment $(8.2 \pm 7.5$ at timepoint 1 vs $7.3 \pm 7.7$ at timepoint 3).

Self-assessment of itch, sleep, and disease severity did not reveal any marked difference between the two patient subcohorts in terms of score reduction (Table 4).

At timepoint 1, AD improvement was experienced by a higher percentage of patients continuing therapy compared to patients discontinuing treatment $(28.8 \%$ vs $15.5 \%, P<0.001)$. Stable AD was reported by $60.9 \%$ of patients continuing therapy compared to $48.6 \%$ of patients interrupting therapy. On the contrary, an increased number of patients discontinuing therapy described worsening of disease compared to patients continuing therapy (35.9\% vs $10.3 \%$ ). Similarly, $A D$ status perceived by patients continuing or interrupting therapy was significantly different at the following timepoints $(P<0.001$; Table 4). Comparing patients treated with dupilumab monotherapy, dupilumab combined with other systemic therapies, and immunosuppressive systemic compounds, a reduction of disease severity (EASI score, and NRS scores) was detected at timepoint 3 vs timepoint 1 , as well as a significantly different AD status across the three patient cohorts at each time point $(P<0.0001$, Table S2). Patients treated with dupilumab monotherapy showed lower disease activity at timepoint 1, with a mean EASI score significantly lower compared to the other patients $(P<0.001)$, and this improvement was sustained thereafter (Table S2).

\section{4 | DISCUSSION}

This observational study included a large population of patients (1831 adult subjects) affected by moderate-to-severe $A D$ and treated with systemic therapies or phototherapy, and managed during the COVID-19 pandemic in Italy. The participating centers $(n=35)$ were highly representative of the different incidence distribution of SARS-CoV-2 infection nationwide, having 15, 10, and 10 centers located in Northern, Central, and Southern Italy, respectively. ${ }^{8}$ During the observation period, a total number of 240,578 SARS-CoV2-positive cases were registered, with a cumulative number of 190,248 recovered cases and 34,767 deaths. ${ }^{8}$ In details, national incidence at timepoint 1,2 , and 3 was $0.71 \%$ (95\% Cl: 0.4-1.2), $0.27 \%$ (95\% Cl: $0.1-0.64)$, and $0.11 \%(95 \% \mathrm{Cl}: 0.0-0.4)$, in line with the infection rate observed in our $A D$ population (timepoint 1 : 0.71\%; timepoint 2: 0.16\%; timepoint 3: 0\%). In our study population, less than $1 \%$ of patients $(16 / 1831)$ resulted positive to SARS-CoV-2, with only three patients who required hospitalization, though swab testing was not massively performed throughout the study period. During this critical sanitary emergency, clinical activity in dermatology clinics was markedly limited, and teledermatology (web- and phone- counseling) was extremely useful for reducing patient access to hospital. This modality was well accepted by AD patients who continued to have access to dermatologist consultation, guaranteeing support and treatment continuation in the majority of cases. Indeed, a relatively low number of patients were lost to follow-up
(7.7\%). As suggested by both national and international scientific societies, most patients were recommended to continue their current treatment during COVID-19 pandemic. ${ }^{2}$ About $86 \%$ of patients continued treatment, including 8 patients who resulted positive to SARS-CoV-2 infection, albeit common recommendations suggested to withdraw therapy. Notably, $85 \%$ of patients included in this study were treated with dupilumab, mostly prescribed as monotherapy.

Considering disease severity assessment, patients undergoing dupilumab monotherapy showed lower disease activity suggesting a better control of $A D$ compared to patients treated with systemic immunosuppressive compounds or dupilumab combined with other systemic therapies. Albeit dupilumab-treated patient cohort exhibited lower disease severity at baseline and throughout the study period compared to the other treatments, superiority of dupilumab during COVID pandemic cannot be suggested based on this data referring to a limited time frame and heterogeneous baseline patients' characteristics across different treatment groups. The therapeutic regimen combining dupilumab with other systemic agents occurred in a cohort of patients with significantly higher prevalence of atopic disorders who may require this combined approach as likely they represent a high-need patient population. Nevertheless, no worsening of atopic comorbid conditions was reported. Response to treatment in these patients resulted similar to patients treated with dupilumab monotherapy or systemic immunosuppressive compounds. This latter class of agents was supposed to have an unfavorable safety profile compared to biologics but no warning signal was detected in our study. Dupilumab does not impair the immune compartments implicated in host defense against viral infections and thus may be considered a safer therapeutic choice for AD..$^{9-12}$ In dupilumab clinical trials, rates of general infections, upper respiratory tract infections, and nasopharyngitis resulted similar to placebo, and, in particular, viral infections, were not reported as meaningful adverse event. ${ }^{7,13}$ In terms of effectiveness, dupilumab therapy obtained a satisfactory control of the disease and consistently with the other systemic compounds, treatment interruption did not cause a rapid and relevant worsening of the disease, as highlighted by the decrease of both patient-assessed severity scores and EASI score in patients discontinuing therapy. This finding is in line with a recent study reporting maintenance of EASI75 response in $30.4 \%$ of high-responding patients treated with dupilumab, after rerandomization to placebo. ${ }^{14}$ However, the reduction of disease severity in patients discontinuing therapy was not associated with a positive patient perception of $A D$ status: a higher percentage of patients withdrawing therapy evaluated their AD status as worsened. Likely, therapy continuation, compared to an intermittent or discontinued therapeutic regimen, might positively impact on patient perception of both disease control and severity.

Dupilumab was interrupted in a small percentage of patients, conversely to cyclosporine and oral corticosteroids. In addition, phototherapy was interrupted in most cases (about 74\%) due to the lack of accessibility to phototherapy services during phase I (lockdown). Dupilumab interruption was mainly based on patient decision, and the main cause of interruption was represented by non-medical reasons (lack of drug supply). Fear of having an increased risk of 
COVID-19 disease determined treatment interruption in 25\% of patients withdrawing therapy, similarly to recent findings observed in psoriasis patients. ${ }^{15}$ Another study confirmed that patients affected by either psoriasis (233 patients) or AD (68 patients) who felt unsafe about their immunomodulatory treatment were more concerned about having SARS-CoV-2 infection and more likely discontinued therapy during pandemic (overall treatment interruption: $7.3 \%)^{16}$ In particular, $A D$ patients with asthma were more concerned about being at risk of COVID-19 disease because of AD and its treatment. ${ }^{16}$

The strength of our study is the large $A D$ population treated with systemic therapies who was observed longitudinally, during the national lockdown period (phase I) and the following phase of partial and gradual re-opening of healthcare services (phase II and III) that were planned in order to face COVID-19 outbreak. In particular, this study provided evidence that continuation of immunomodulant/immunosuppressive therapies during COVID-19 pandemic can be considered safe and effective in controlling AD. This finding strengthens the recommendations issued by national and international scientific societies at the beginning of the COVID-19 outbreak that are based on experts' opinion. ${ }^{2-5}$ Notably, this study also suggested that drug interruption did not cause AD flares, as treatment response was maintained in the short term.

However, some limitations related to the data collection, management, and disease severity evaluation via web or phone counseling should be considered as most of the assessment tools used were patient-reported and only a minor percentage of patients could be evaluated by regular visits during phase 3 . Detailed information about atopic comorbid conditions, SARS-CoV-2 serology testing were not collected. In addition, most patients were undergoing dupilumab therapy with a satisfactory control of the disease, particularly with dupilumab monotherapy (mean T1 EASI score significantly lower than other treatment groups), and this could represent a selection bias of the study population likely related to the relatively higher number of dupilumab-treated patients managed in a dedicated AD outpatient clinic.

Data collection related to $A D$ patients treated with systemic compounds and/or phototherapy during COVID-19 pandemic is continuing by the DA-COVID-19 registry, willing to delineate the infectious risk related to the use of each immunomodulant/immunosuppressant agent in $A D$ patient population and to better characterize COVID-19 outcomes in patients with AD, as internationally promoted by the SECURE-AD registry. ${ }^{17}$

\section{ACKNOWLEDGMENTS}

A. Chiricozzi, M. Talamonti, M. Galluzzo, and K. Peris conceptualize this study, analyzed data, and wrote the manuscript. A. Chiricozzi, N. Gori, L. Di Nardo, F. Guarneri performed and verified statistical analyses. G. Girolomoni, L. Stingeni, P. Amerio, F. Guarneri, C. De Simone, A. Patrizi, A. Belloni Fortina, F. Rongioletti, G. Fabbrocini, A.V Marzano, G. Micali, A. Offidani, M.T. Rossi, L. Bianchi, A. Cristaudo, M.T. Fierro, G. Pellacani, G. Argenziano, P. Pigatto, M. Romanelli, P. Savoia, P. Rubegni, C. Foti, N. Milanesi, M.R. Bongiorno, T. Grieco, S. Di Nuzzo, M.C. Fargnoli, A. Carugno, A. Motolese, R. Balestri, C. Potenza, C. Patruno, I. Zalaudek, M. Lombardo, and C. Feliciani contributed to both data collection and the interpretation of the results.
The collaborators included in the DA-COVID-19 group contributed in managing patients during the study period. All authors revised the manuscript and approved the submitted version.

\section{CONFLICTS OF INTEREST}

A. Chiricozzi served as advisory board member and consultant and has received fees and speaker's honoraria or has participated in clinical trials for AbbVie, Almirall, Biogen, Fresenius Kabi, Leo Pharma, Lilly, Janssen, Novartis, Sanofi Genzyme, and UCB Pharma. G. Fabbrocini acted as speaker and consultant for AbbVie and Leo Pharma. G. Girolomoni has been principal investigator in clinical trials sponsored by and/or and has received personal fees from AbbVie, Abiogen, Almirall, Amgen, Biogen, Boehringer-Ingelheim, Bristol-Meyers Squibb, Celgene, Celltrion, Eli-Lilly, Genzyme, Leo Pharma, Menlo therapeutics, Novartis, OM Pharma, Pfizer, Regeneron, Samsung, Sandoz, and UCB Pharma. A. Offidani has been a scientific consultant/speaker/clinical study investigator for AbbVie, Celgene, Janssen, LEO Pharma, Eli-Lilly, MSD, Novartis, Pfizer, Sanofi, Alfasigma, and Almirall. M.T. Rossi has received personal fee for advisory board meeting from Sanofi, Abbvie, Novartis, and Cantabria. L. Bianchi reports personal fees from speaker and as consultant for AbbVie, Novartis, Janssen-Cilag, Pfizer, UCB, and Leo Pharma, outside the submitted work. L. Stingeni has been principal investigator in clinical trials sponsored by and/or received personal fees from AbbVie, Almirall, Celgene, Eli-Lilly, Janssen, Novartis, and Sanofi-Genzyme. G. Pellacani has been principal investigator in clinical trials sponsored by and/or received personal fees from AbbVie, Almirall, Eli-Lilly, Leo Pharma, Novartis, and Sanofi. A. Patrizi has served as a speaker and received honoraria from Sanofi-Genzyme for lectures, research grants and as an advisory board member. C. Foti has been speaker for Sanofi and Abbvie. M.C. Fargnoli has served on advisory boards, received honoraria for lectures and research grants from Almirall, AbbVie, Galderma, Leo Pharma, Mylan, Medac Pharma, Celgene, Pierre Fabre, UCB, Eli-Lilly, Pfizer, Janssen, Novartis, Sanofi Genzyme, Roche, Sun Pharma, and MSD. F. Rongioletti has served on advisory board, received honoraria for lectures and research grants from Novartis, AbbVie, Janssen-Cilag, Eli-Lilly, Leo Pharma, and Sanofi-Genzyme. P. Amerio has received speaker honoraria from Sanofi, AbbVie, Janssen, Celgene, Novartis, and Sandoz. G. Micali has been a scientific consultant/clinical study investigator for Abbvie, Eli-Lilly, Janssen- Cilag, LEO Pharma, and Novartis. C. Patruno has been a consultant and held sponsored conferences for AbbVie, Novartis, Pfizer, and Sanofi. I. Zalaudek has been a consultant and/or speaker for Novartis, Celgene, and Amgen. $K$. Peris reports grants and personal fees for advisory board meeting from Almirall, AbbVie, Biogen, Lilly, Celgene, Galderma, Leo Pharma, Novartis, Pierre Fabre, Sanofi, Sandoz, Sun Pharma, and Janssen. The other authors reported no conflicts of interests.

\section{ORCID}

Andrea Chiricozzi (D) https://orcid.org/0000-0002-6739-0387

Luca Stingeni (D) https://orcid.org/0000-0001-7919-8141

Andrea Carugno (D) https://orcid.org/0000-0002-8231-2205 


\section{REFERENCES}

1. Data on SARS-CoV-2 pandemic worldwide provided by the World Health Organization. https://www.who.int/docs/default-sourc e/coronaviruse/transcripts/who-audio-emergencies-coronaviru s-press-conference-full-and-final-11mar2020.pdf?sfvrsn=cb432 bb3_2

2. Wollenberg A, Flohr C, Simon D, et al. European Task Force on Atopic Dermatitis statement on severe acute respiratory syndrome coronavirus 2 (SARS-Cov-2) infection and atopic dermatitis. J Eur Acad Dermatol Venereol 2020;34:e241-e242.

3. Vultaggio A, Agache I, Akdis CA, et al. Considerations on biologicals for patients with allergic disease in times of the COVID-19 pandemic: an EAACI Statement [published online ahead of print, 2020 Jun 5]. Allergy. 2020; https://doi.org/10.1111/all.14407

4. National Recommendations by the Italian Society of Dermatology. https://www.sidemast.org/blog/coronavirus

5. Shah M, Sachdeva M, Alavi A, Shi VY, Hsiao JL. Optimizing care for atopic dermatitis patients during the COVID-19 pandemic. J Am Acad Dermatol. 2020;8:e165-e167.

6. Giuliani F, Gualdi G, Amerio P. Effect of immunosuppressive drugs in immune-mediated inflammatory disease during the coronavirus pandemic. Dermatol Ther. 2020;e14204.

7. Kearns DG, Uppal S, Chat VS, Wu JJ. Assessing the risk of dupilumab use for atopic dermatitis during the COVID-19 pandemic. J Am Acad Dermatol. 2020;83:e251-e252.

8. Data related to COVID-19 pandemic in Italy http://opendatadpc.maps.arcgis.com/apps/opsdashboard/index.html\#/ b0c68bce2cce478eaac82fe38d4138b1.

9. Napolitano M, Patruno C, Ruggiero A, Nocerino M, Fabbrocini G. Safety of dupilumab in atopic patients during COVID-19 outbreak. J Dermatolog Treat. 2020;1-2.

10. Ferrucci S, Romagnuolo M, Angileri L, Berti E, Tavecchio S. Safety of dupilumab in severe atopic dermatitis and infection of Covid-19: two case reports. J Eur Acad Dermatol Venereol. 2020;34:e303-e304

11. Caroppo F, Biolo G, Belloni FA. SARS-CoV-2 asymptomatic infection in a patient under treatment with dupilumab. J Eur Acad Dermatol Venereol. 2020;34:e368.

\section{APPENDIX 1}

\section{DA-COVID-19 STUDY GROUP}

Giacomo Caldarola, Dermatologia, Dipartimento Scienze Mediche e Chirurgiche, Fondazione Policlinico Universitario A. Gemelli IRCCS, Rome, Italy; Dionisio Silvaggio, Dermatology Unit, Policlinico Tor Vergata, Department of Systems Medicine, Tor Vergata University of Rome, Italy; Annunziata Dattola, Dermatology Unit, Policlinico Tor Vergata, Department of Systems Medicine, Tor Vergata University of Rome, Italy; Maddalena Napolitano, Section of Dermatology Department of Clinical Medicine and Surgery, University of Naples Federico II, Naples, Italy; Silvia Mariel Ferrucci, Dermatology Unit, Fondazione IRCCS Ca' Granda Ospedale Maggiore Policlinico, Milan, Italy; Giacomo Dal Bello, Section of Dermatology and Venereology, Department of Medicine, University of Verona, Verona, Italy Tommaso Bianchelli, Dermatological Clinic, Department of Clinica and Molecular Sciences, Polytechnic University of the Marche Region, Ancona, Italy; Chiara Rovati, Department of Dermatology, ASST Spedali Civili of Brescia, University of Brescia, Brescia, Italy; Flavia Pigliacelli, Clinical Dermatology, San Gallicano Dermatological Institute, Rome, Italy; Michela Ortoncelli, Medical Sciences
12. Carugno A, Raponi F, Locatelli AG, et al. No evidence of increased risk for COVID-19 infection in patients treated with Dupilumab for atopic dermatitis in a high-epidemic area - Bergamo, Lombardy, Italy. J Eur Acad Dermatol Venereol 2020; https://doi.org/10.1111/jdv.16552

13. Eichenfield LF, Bieber T, Beck LA, et al. Infections in dupilumab clinical trials in atopic dermatitis: a comprehensive pooled analysis. Am J Clin Dermatol. 2019;20:443-456.

14. Worm M, Simpson EL, Thaçi D, et al. Efficacy and safety of multiple dupilumab dose regimens after initial successful treatment in patients with atopic dermatitis: a randomized clinical trial. JAMA Dermatol. 2020;156:131-143.

15. Pirro F, Caldarola G, Chiricozzi A, et al. The impact of COVID-19 pandemic in a cohort of Italian psoriatic patients treated with biological therapies. J Dermatolog Treat. 2020;1-5.

16. Dyrberg Loft N, Halling AS, Iversen L, et al. Concerns related to the COVID-19 pandemic in adult patients with atopic dermatitis and psoriasis treated with systemic immunomodulatory therapy: a Danish questionnaire survey. J Eur Acad Dermatol Venereol. 2020; https://doi.org/10.1111/jdv.16863

17. Mahil SK, Yiu ZZN, Mason KJ, et al. Global reporting of cases of COVID-19 in psoriasis and atopic dermatitis: an opportunity to inform care during a pandemic. Br J Dermatol. 2020;183:404-406.

\section{SUPPORTING INFORMATION}

Additional supporting information may be found online in the Supporting Information section.

How to cite this article: Chiricozzi A, Talamonti M, De Simone C, et al. Management of patients with atopic dermatitis undergoing systemic therapy during COVID-19 pandemic in Italy: Data from the DA-COVID-19 registry. Allergy. 2021;00:1-12. https://doi.org/10.1111/all.14767

Department, Dermatologic Clinic, University of Turin, Turin, Italy Katharina Hansel, Dermatology Section, Department of Medicine, University of Perugia, Perugia, Italy; Giulia Calabrese, Dermatology Unit, University of Campania Luigi Vanvitelli, Naples, Italy; Camilla Loi, Dermatology UOC, Department of Experimental, Diagnostic and Specialty Medicine, University of Bologna, Italy; Michela lannone, Department of Dermatology, University of Pisa, Pisa, Italy; Federica Veronese, Department of Health Sciences, Amedeo Avogadro University of Eastern Piedmont, Novara, Italy; Paolo Romita, Department of Biomedical Science and Human Oncology, Unit of Dermatology, University of Bari, Bari, Italy; Greta Tronconi, Dermatology Clinic, Department of Health Sciences, University of Florence, Florence, Italy; Francesca Caroppo, Dermatology Unit - Department of Medicine DIMED, University of Padova, Padova, Italy; Giovanna Tilotta, Section of Dermatology, Department of Health Promotion, Mother and Child Care, Internal Medicine and Medical Specialties, University of Palermo, Palermo, Italy; Alvise Sernicola, Unit of Dermatology, Department of Clinical Internal, Anesthesiological and Cardiovascular Sciences, Sapienza University of Rome, Rome, Italy; Maria Esposito, Dermatology, Department of Biotechnological and Applied Clinical Sciences, University of L'Aquila, 
L'Aquila, Italy; Francesca Raponi, Dermatology Unit, ASST Papa Giovanni XXIII Hospital, Bergamo, Italy; Giulio Gualdi, Department of Medicine and Aging Science, Dermatologic Clinic, G. D'Annunzio University, Chieti, Italy; Giulia Rech, Division of Dermatology, Santa Chiara Hospital, Trento, Italy; Maria Letizia Musumeci, Dermatology Unit, Department of Health Sciences, Università Magna Graecia, Catanzaro, Italy; Steven Paul Nisticò, Dermatology Unit, Department of Health Sciences, Università Magna Graecia, Catanzaro, Italy; Alessio Campitiello, Dermatology, Department of Clinical and Experimental Medicine, University of Messina, Messina, Italy; Laura Bonzano, Allergology, ASL Modena, Italy; Viviana Piras, Section of Dermatology, Department of Medical Sciences and Public Health, University of Cagliari, Cagliari, Italy. 